\title{
Multicopy Vectors Carrying the Klebsiella pneumoniae nifA Gene Do Not Enhance the Nodulation Competitiveness of Sinorhizobium meliloti on Alfalfa
}

\author{
Pieter van Dillewijn, Francisco Martínez-Abarca, and Nicolás Toro \\ Departamento de Microbiología del Suelo y Sistemas Simbióticos, Estación Experimental del Zaidín, Con- \\ sejo Superior de Investigaciones Cientificas, Calle Profesor Albareda1, 18008 Granada, Spain \\ Accepted 22 April 1998.
}

\begin{abstract}
It has been reported that Sinorhizobium meliloti strains harboring IncQ and IncP multicopy vectors containing constitutively expressed Klebsiella pneumoniae nifA exhibit an increase in nodulation competitiveness on alfalfa (J. Sanjuan and J. Olivares, Mol. Plant-Microbe Interact. 4:365-369, 1991). In our efforts to understand the mechanisms involved, in this work, we have found that the observed enhancement on nodulation competitiveness by IncQ derivatives carrying $K$. pneumoniae nifA was not dependent on the plasmid-borne nif $A$ activity but on the sensitivity of nonresistant strains to the streptomycin carried over from growth cultures. Furthermore, it was also determined that the nifA of $K$. pneumoniae on an IncP vector does not have an effect on competitiveness.
\end{abstract}

Leguminous crops have been inoculated with Rhizobium and Bradyrhizobium spp. since the end of the last century with the aim to improve legume yields. However, the development of highly efficient rhizobial inoculants has been hampered by the inability of the released strains to compete for legume nodulation against the well-adapted indigenous population. This is known as "the competitiveness problem," which has been extensively reviewed (Maier and Triplett 1996; Toro 1996). The competitive ability of rhizobial strains is affected by many environmental and genetic factors that complicate efforts to overcome this problem. Nevertheless, one strategy that could be pursued is to introduce genes that give the recipient bacteria an advantage over the indigenous population. Some genes of interest include those that produce and confer resistance to antibiotics such as trifolitoxin (Robleto et al. 1997), or those involved in improving nodulation efficiency, such as the nfe genes (Soto et al. 1994; Chun and Stacey 1994). Additionally, genes that give a metabolic advantage such as rhizopine synthesis and catabolism genes ( $\mathrm{mos} / \mathrm{moc}$ ) (Murphy et al. 1995) or the proline dehydrogenase putA gene (Jiménez-Zurdo et al. 1995), could be considered. Finally, the regulatory gene nifA has been proposed to play a role in competitiveness in Sinorhizobium meliloti (Sanjuan and Olivares

Corresponding author: Nicolás Toro; Telephone: + 34 (9) 58 121011; Fax: + 34 (9) 58 129600; E-mail: ntoro@eez.csic.es
1991). Sanjuan and Olivares (1991) reported that multicopy plasmids (pCK1 and pCK3) containing the nifA gene of Klebsiella pneumoniae enhance the competitive ability of $S$. meliloti strains. These authors hypothesized that this positive effect on $S$. meliloti nodulation competitiveness might be explained by the presence of nifA-regulated, $n f e$-analogous genes. More recently, Herrera-Cervera et al. (1997) reported that the reduced nodulation competitiveness shown by an $S$. meliloti GR4 $\mathrm{RecA}^{-}$derivative strain could be enhanced by the presence of plasmid pCK3. Furthermore, it has been reported that pCK3 in S. meliloti has a positive effect on mycorrhiza formation (Tobar et al. 1996). The mechanism by which these multicopy vectors carrying $K$. pneumoniae nifA enhance $S$. meliloti competitiveness and mycorrhiza formation remains unknown. Therefore, the potential use (if any) of $K$. pneumoniae nifA gene as a genetic tool to enhance the competitiveness of $S$. meliloti still needs to be elucidated.

Here, in contradiction to published results (Sanjuan and Olivares 1991; Herrera-Cervera et al. 1997), we report that multicopy vectors carrying $K$. pneumoniae nifA do not enhance the nodulation competitiveness of $S$. meliloti on alfalfa.

Bacterial strains and plasmids used and constructed in this work are listed and described in Table 1. Escherichia coli and $S$. meliloti strains were routinely grown as described by Jiménez-Zurdo et al. (1995). Antibiotics were used as required at the following concentrations $\left(\mu \mathrm{g} \mathrm{ml}^{-1}\right)$ : tetracycline (10), ampicillin (200), kanamycin (50 for E. coli and 180 for Sinorhizobium strains), spectinomycin (100), streptomycin (50 for $E$. coli and 250 for Sinorhizobium strains), chloramphenicol (50), and gentamycin (30).

To test the effect of $K$. pneumoniae nifA on S. meliloti competitiveness, plasmid pDIL120, an analogous construction to pCK1, was made by cloning the wild-type nifA gene, preceded by a constitutive kanamycin resistance gene promoter, into the IncQ plasmid pMP77 (Fig. 1 and Table 1). As a control, a frameshift mutation was induced in the nifA gene to give pDIL119 (Fig. 1 and Table 1). The nifA activity produced by these constructions was determined with a plasmid carrying a $\mathrm{P} 1$ (nifH promoter)-lacZ fusion as a reporter in $E$. coli cells, as described by Better et al. (1985). The results indicated that the nifA activity exhibited by pDIL120 is similar to that of pCK1 while pDIL119 lacks this activity (data not shown). 
Competitive assays were performed with $S$. meliloti strains containing the different constructions. Alfalfa (Medicago sativa L.) plants were grown under axenic conditions in nitrogen-free nutrient solution as described by Olivares et al. (1980). For competition assays, 10-day-old plants (10 to 12 tubes) were inoculated with $10^{5}$ bacteria $\mathrm{ml}^{-1}$ each. Prior to inoculation, rhizobia were grown to exponential phase $\left(\mathrm{OD}_{600} 0.4\right.$ to 0.6$)$ in TY medium supplemented with antibiotics where appropriate for plasmid maintenance. To obtain the $1: 1$ ratio required in the coinoculation mixture, strains were diluted (1,000-fold) together from their respective cultures after both had reached the same optical density. To distinguish between strains occupying nodules, one of the strains of the coinoculation mixture was marked with the $\beta$-glucuronidase (GUS) gene. $S$. meliloti strains GR4 and GRM6 were marked with the construction pGUS3, while the 2011 derivative strain, M4, contains the GUS gene incorporated into its chromosome (Table 1). The plants were collected 14 to 15 days after inoculation and the roots briefly washed with water. Roots were incubated in darkness overnight at $37^{\circ} \mathrm{C}$ in $1 \mathrm{mM} \mathrm{X}$ Gluc (5-bromo-4-chloro-3-indolyl- $\beta$-D-glucuronide; Apollo Scientific, Stockport, UK) in $50 \mathrm{mM}$ sodium-phosphate buffer ( $\mathrm{pH} 7.5)$ with sodium dodecyl sulfate $1 \%$ (wt/vol). Nodule occupancy was determined by counting blue and white nodules. As shown in Table 2, all the strains carrying IncQ derivative plasmids, including the original vectors for pCK1 and pDIL120, namely, RSF1010 and pMP77, respectively, as well as the strain carrying pDIL119 in which nifA had been inactivated, occupied $100 \%$ of the alfalfa root nodules. Similar results were previously reported (Sanjuan 1989; Sanjuan and Olivares 1991) with the plasmid pCK1 in different S. meliloti strains. However, when the bacteria were washed prior to inoculation, nodulation occupancy by both the $S$. meliloti strains carrying pDIL119 or pDIL120 decreased to about 50\% (Table 2, washed samples). These results clearly indicate that the nifA gene on IncQ derivative plasmids is not involved in enhancing the competitiveness of $S$. meliloti strains.

In the search for a possible explanation, the cell viability of GRM6(pGUS3) and GRM6(RSF1010) was checked when coinoculated in hydroponic culture with or without alfalfa present. While GRM6 carrying RSF1010 maintains growth until a plateau is reached at about $10^{7}$ cells $\mathrm{ml}^{-1}$, the viability of GRM6 carrying pGUS3 decreased to $10^{2}$ cells ml $\mathrm{ml}^{-1}$ in the first day after inoculation and became undetectable after 5 days. Although the presence of an alfalfa plant appeared to reduce the sensitivity of GRM6 carrying pGUS3, the initial $1: 1$ ratio still dropped by 1,000 -fold after 5 days of inocula-
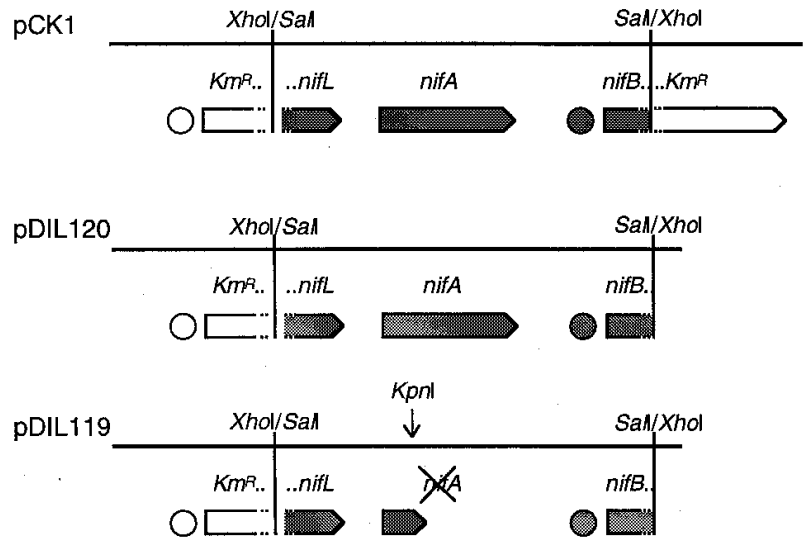

Fig. 1. Comparison of the nifA DNA region in pCK1, pDIL120, and pDIL119. In all three constructions, a SalI fragment containing the Klebsiella pneumoniae nifA and flanking fragments of nifL and nifB was cloned into the XhoI site of the kanamycin $(\mathrm{Km})$ resistence gene. The indicated $K p n \mathrm{I}$ site within nifA was used for constructing the frameshift mutation in pDIL119 to give a truncated open reading frame ending 23 amino acids after the mutation site. Promoters are indicated by circles.

Table 1. Bacterial strains and plasmids

\begin{tabular}{|c|c|c|}
\hline Strains / plasmids & Relevant characteristics & Source or reference \\
\hline \multicolumn{3}{|l|}{ Escherichia coli } \\
\hline DH5 $\alpha$ & recA endA1 gyrA96 thi-1 hsdR17 supE44 $\Delta l a c U 169(\Phi 80 \mathrm{~d} l a c Z \Delta m 15)$ deoR $F^{-} \lambda$ & Bethesda Research Laboratory \\
\hline \multicolumn{3}{|c|}{ Sinorhizobium meliloti } \\
\hline GR4 & Wild-type, $\mathrm{Nod}^{+}, \mathrm{Fix}^{+}$ & This laboratory \\
\hline GRM6 & GR4 derivative cured of cryptic plasmid pRmeGR4b & Mercado-Blanco and Olivares 1993 \\
\hline 2011 & SU47 derivative strain, $\mathrm{Nod}^{+}, \mathrm{Fix}^{+}$ & J. Denarié \\
\hline M4 & $\begin{array}{l}2011 \text { with gus gene integrated into a nonessential region betweeen alaS and } \operatorname{rec} A \text { in the } \\
\text { chromosome, } \mathrm{Sm}^{\mathrm{r}}\end{array}$ & Selbitschka et al. 1995 \\
\hline \multicolumn{3}{|c|}{ 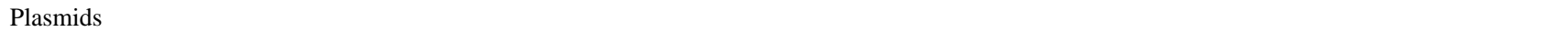 } \\
\hline $\mathrm{pIC} 20 \mathrm{H}$ & Cloning vector, $\mathrm{Ap}^{\mathrm{r}}$ & Marsh et al. 1984 \\
\hline $\mathrm{pJB} 3 \mathrm{KmD}$ & Broad host range IncP cloning vector, $6052 \mathrm{bp}, \mathrm{Ap}^{\mathrm{r}}, \mathrm{Km}^{\mathrm{r}}$ & J. M. Blatny \\
\hline pMP77 & Broad host range IncQ cloning vector, derivative of pKT214, $x y l E, \mathrm{Cm}^{\mathrm{r}}, \mathrm{Sm}^{\mathrm{r}}$ & H. P. Spaink \\
\hline pKT214 & Broad host range IncQ cloning vector, derivative of RSF1010, $\mathrm{Tc}^{\mathrm{r}}, \mathrm{Cm}^{\mathrm{r}}, \mathrm{Sm}^{\mathrm{r}}$ & Bagdasarian et al. 1979 \\
\hline pRK290 & Broad host range IncP cloning vector, $\mathrm{Tc}^{\mathrm{r}}$ & Ditta et al. 1980 \\
\hline RSF1010 & Broad host range IncQ cloning vector, $\mathrm{Sm}^{\mathrm{r}}$ & Guerry et al. 1974 \\
\hline pGUS3 & $n f e D$ promoter-GUS fusion on $\mathrm{pBI} 101$ (Clontech), $\mathrm{Km}^{\mathrm{r}}$ & F. M. Garcia-Rodriguez \\
\hline pCK1 & RSF1010 derivative containing the nifA of Klebsiella pneumoniae, $\mathrm{Sm}^{\mathrm{r}}, \mathrm{Km}^{\mathrm{r}}$ & Kennedy and Robson 1983 \\
\hline $\mathrm{pCK} 3$ & pRK290 derivative containing the nifA of $K$. pneumoniae, $\mathrm{Tc}^{\mathrm{r}}$ & C. Kennedy \\
\hline pWK23 & Cloning vector containing nif $L A$ and nifBQ of $K$. pneumoniae, $\mathrm{Tc}^{\mathrm{r}}$ & Arnold et al. 1988 \\
\hline pDIL15 & Kanamycin cassette cloned as a $P$ stI fragment from $\mathrm{pJB} 3 \mathrm{KmD}$ into $\mathrm{pIC} 20 \mathrm{H}, \mathrm{Ap}^{\mathrm{r}}, \mathrm{Km}^{\mathrm{r}}$ & This study \\
\hline pDIL19 & $\begin{array}{l}\text { pDIL20 with disruption of the nifA gene by frameshift mutation created by cutting with } \\
\text { KpnI and filling-in, } \mathrm{Ap}^{\mathrm{r}}\end{array}$ & This study \\
\hline pDIL20 & $\begin{array}{l}\text { XhoI fragment of pDIL } 15 \text { containing most of the kanamycin resistant gene was replaced } \\
\text { by the SalI fragment of pWK23 containing nifA, } \mathrm{Ap}^{\mathrm{r}}\end{array}$ & This study \\
\hline pDIL119 & HindIII fragment containing nifA of pDIL19 cloned into pMP77, $\mathrm{Cm}^{\mathrm{r}}, \mathrm{Sm}^{\mathrm{r}}$ & This study \\
\hline pDIL120 & HindIII fragment containing nifA of pDIL20 cloned into pMP77, $\mathrm{Cm}^{\mathrm{r}}, \mathrm{Sm}^{\mathrm{r}}$ & This study \\
\hline
\end{tabular}


tion. On the other hand, when the bacterial cells were washed prior to coinoculation, the cell viability of both GRM6 carrying pGUS3 and GRM6 carrying RSF1010 was similarly unaffected (data not shown).

The antibiotic streptomycin is used to grow $S$. meliloti strains carrying IncQ derivative plasmids to ensure its maintenance. When the bacteria are coinoculated without washing prior to inoculation, the antibiotic streptomycin reaches a final concentration of $250 \mathrm{ng} \mathrm{ml}^{-1}$ (1,000-fold dilution) in the hydroponic culture. We tested whether this amount of streptomycin affects the cell viability of GRM6(pGUS3) in the hydroponic culture. As shown in Figure 2, the number of viable cells of this strain decreases dramatically in the first day after inoculation although, thereafter, the surviving bacteria apparently begin to increase their number. This recovery of bacterial cell growth of the sensitive strain was not observed in the competition assays described above, which might be explained by the presence of a growing resistant competitor

Table 2. Competitive assays with IncQ derivatives in Sinorhizobium meliloti

\begin{tabular}{|c|c|c|}
\hline Inoculum & $\begin{array}{c}\text { White } \\
\text { nodules }(\%)^{\mathrm{a}}\end{array}$ & $\begin{array}{c}\text { Nodules } \\
\text { (total no.) }\end{array}$ \\
\hline GR4(pGUS3) × GR4(pCK1) & 100 & 266 \\
\hline GRM6(pGUS3) × GRM6(pCK1) & 100 & 271 \\
\hline GRM6(pGUS3) × GRM6(RSF1010) & 100 & 288 \\
\hline GRM6(pGUS3) $\times$ GRM6 $($ pMP77) & 100 & 260 \\
\hline GRM6(pGUS3) × GRM6(pDIL119) & 100 & 264 \\
\hline GRM6(pGUS3) $\times$ GRM6(pDIL120) & 100 & 298 \\
\hline GRM6 $($ pGUS3 $) \times$ GRM6(pDIL119) ${ }^{b}$ & $55.3 \pm 6.2$ & 246 \\
\hline GRM6(pGUS3) $\times$ GRM6(pDIL120) ${ }^{b}$ & $42.9 \pm 6.3$ & 238 \\
\hline
\end{tabular}

a Nodules occupied by bacteria containing pGUS3 (single and double occupancy) stain blue. Error values are indicated at a $95 \%$ confidence interval

${ }^{\mathrm{b}}$ Washed prior to inoculation.



Fig. 2. Effect of diluted TY with or without streptomycin on viability of GRM6(pGUS3) cells in hydroponic medium (final concentration of streptomycin when present is $250 \mathrm{ng} \mathrm{m}^{-1}$ ). GRM6(pGUS3) coinoculated with only TY medium (open circles) or with TY medium containing streptomycin (black boxes). Sinorhizobium meliloti cell viability was analyzed during 5 days by taking $0.1-\mathrm{ml}$ samples of inoculated media and plating corresponding dilutions on plates containing TY medium with kanamycin. Values represent means of three independent experiments. Error bars show corresponding standard errors. Where no bars are seen error is small. strain and the consequent depletion of available carbon and nitrogen sources. Contrary to the results obtained with streptomycin, the 1,000-fold dilution of other antibiotics routinely used in our assays (tetracycline, ampicillin, kanamycin, spectinomycin, chloramphenicol, and gentamycin) showed no effect on cell viability (data not shown). Therefore, the nodule occupancy by $S$. meliloti strains carrying IncQ derivative plasmids appears to be a consequence of the sensitivity of the other strains in the coinoculation mixture to streptomycin carried over from the cultures of the $S$. meliloti strains bearing IncQ plasmid derivatives.

The effect of streptomycin carry-over on nodule occupancy should be eliminated either by washing cells prior to coinoculation or by coinoculation with both partners being resistant to this antibiotic. The latter is the case for M4, an $S$. meliloti 2011 derivative containing the GUS gene within the chromosome (Selbitschka et al. 1995), and 2011 carrying either pCK1 or RSF1010. The results presented in Table 3 with these strains confirm those obtained in Table 2 (washed samples). Once more, the strain containing the $K$. pneumoniae nifA (pCK1) not only failed to enhance competitiveness when compared with the strain containing RSF1010 or the control (M4 washed $\times 2011$ ), but even reduced the number of nodules occupied by the carrying strain. Likewise, the comparison between washed and unwashed M4 against 2011 confirmed the effect of low concentrations of streptomycin in the results of the competitive assays.

Sanjuan and Olivares (1991) also reported that the IncP derivative plasmid pCK3 carrying $K$. pneumoniae nifA improved the competitive ability of $S$. meliloti strain 2011 . This plasmid encodes for kanamycin resistance and therefore the effect on nodulation competitiveness cannot be attributed to the carryover of antibiotic. We introduced plasmid pCK3 and the original vector pRK290 into strain M4 and performed competitive assays. The results shown at the bottom of Table 3 indicate that the presence of neither pCK3 nor pRK290 give significant differences in nodule occupation when compared with the wild-type strain. This implies that, as with IncQ vectors, the nifA of $K$. pneumoniae on an IncP vector does not have an effect on competitiveness.

From the data presented in this report we conclude that multicopy vectors carrying $K$. pneumoniae nifA do not enhance $S$. meliloti nodulation competitiveness on alfalfa.

\section{ACKNOWLEDGMENTS}

We would like to thank Herman P. Spaink for providing vectors pMP77 and pIC20H, Ramón Díaz for RSF1010, Walter Arnold for

Table 3. Competition assays with Sinorhizobium meliloti strain 2011

\begin{tabular}{|c|c|c|}
\hline Inoculum & White nodules $(\%)^{\mathrm{a}}$ & Nodules (total no.) \\
\hline $\mathrm{M} 4^{\mathrm{b}} \times 2011$ & $39.2 \pm 4.1$ & 554 \\
\hline M4 × 2011 & $1.3 \pm 1.1$ & 393 \\
\hline M4 × 2011(RSF1010) & $26.7 \pm 6.0$ & 208 \\
\hline M4 × 2011(pCK1) & $8.7 \pm 3.4$ & 263 \\
\hline M4(pRK290) $)^{\mathrm{b}} \times 2011$ & $47.5 \pm 5.8$ & 284 \\
\hline $\mathrm{M} 4(\mathrm{pCK} 3)^{\mathrm{b}} \times 2011$ & $37.4 \pm 5.7$ & 273 \\
\hline
\end{tabular}

a Nodules occupied by bacteria containing the 2011 strain marked with gus, M4 (single and double occupancy), stain blue. Error values are indicated at a $95 \%$ confidence interval.

${ }^{\mathrm{b}}$ Washed prior to inoculation. 
pWK23, and Werner Selbitschka for strain M4. Furthermore, we thank María José Soto for critical reading of this manuscript and helpful comments. This work was supported by Comisión Asesora de Investigación Científica y Técnica Grant BIO96-0397

\section{LITERATURE CITED}

Arnold, W., Rump, A., Klipp, W, Priefer, U. B., and Pühler, A. 1988. Nucleotide sequence of a 24206-base pair DNA fragment carrying the entire nitrogen fixation cluster of Klebsiella pneumoniae. J. Mol. Biol. 203:715-738.

Bagdasarian, M., Bagdasarian, M. M., Coleman, S., and Timmis, K. N 1979. New vector plasmids for gene cloning in Pseudomonas. Pages 411-422 in Plasmids of Medical, Environmental and Commercial Importance. K. N. Timmis and A. Pühler, eds. Elsevier/North-Holland Biomedical Press, Amsterdam.

Better, M., Ditta, G., and Helinski, D. R. 1985. Deletion analysis of Rhizobium meliloti symbiotic promoters. EMBO J. 4:2419-2424.

Chun, J.-Y., and Stacey, G. 1994. A Bradyrhizobium japonicum gene essential for nodulation competitiveness is differentially regulated from two promoters. Mol. Plant-Microbe Interact. 7:248-255.

Ditta, G., Stanfield, S., Corbin, D., and Helinski, D. R. 1980. Broad host range DNA cloning system for Gram-negative bacteria: Construction of a gene bank of Rhizobium meliloti. Proc. Natl. Acad. Sci. USA 77: 7347-7351

Guerry, P., van Embden, J., and Falkow, S. 1974. Molecular nature of two nonconjugative plasmids carrying drug resistance genes. J. Bacteriol. 117:619-630.

Herrera-Cervera, J. A., Rodriguez-Alonso, F. I., Olivares, J., and Sanjuan, J. 1997. Evaluation of the recA-based containment system in Rhizobium meliloti GR4. FEMS Microbiol. Ecol. 22:49-52.

Jiménez-Zurdo, J. I., van Dillewijn, P., Soto, M. J., de Felipe, M. R., Olivares, J., and Toro, N. 1995. Characterization of a Rhizobium meliloti proline dehydrogenase mutant altered in nodulation efficiency and competitiveness on alfalfa roots. Mol. Plant-Microbe Interact. 8:492-498.

Kennedy, C., and Robson, C. L. 1983. Activation of nif gene expression in Azotobacter by nifA gene product of Klebsiella pneumoniae. Nature 301:626-628.
Maier, R. J., and Triplett, E. W. 1996. Toward more productive, efficient, and competitive nitrogen-fixing symbiotic bacteria. Crit. Rev. Plant Sci. 15:191-234

Marsh, J. L., Erfle, M., and Wykes, E. J. 1984. The pIC plasmid and phage vectors with versatile cloning sites for recombinant selection by insertional inactivation. Gene 32:481-485.

Mercado-Blanco, J., and Olivares, J. 1993. Stability and transmissibility of the cryptic plasmids of Rhizobium meliloti GR4. Arch. Microbiol. 160:477-485

Murphy, P. J., Wexler, W., Grzemski, W., Rao, J. P., and Gordon, D. 1995. Rhizopines - their role in symbiosis and competition. Soil Biol. Biochem. 27:525-529.

Olivares, J., Casadesús, J., and Bedmar, E. J. 1980. Method for testing degree of infectivity of Rhizobium meliloti strains. Appl. Environ. Microbiol. 39:967-970.

Robleto, E. A., Scupham, A. J., and Triplett, E. W. 1997. Trifolitoxin production in Rhizobium etli strain CE3 increases competitiveness for rhizosphere colonization and root nodulation of Phaseolus vulgaris in soil. Mol. Plant-Microbe Interact 10:228-233.

Sanjuan, J. 1989. Identificacion de genes regulados por nifA que afectan la infectividad y competitividad de Rhizobium meliloti. Ph.D. thesis. University of Granada, Granada, Spain.

Sanjuan, J., and Olivares, J. 1991. Multicopy plasmids carrying the Klebsiella pneumoniae nifA gene enhance Rhizobium meliloti nodulation competitiveness on alfalfa. Mol. Plant-Microbe Interact. 4:365-369.

Selbitschka, W., Dresing, U., Hagen, M., Niemann, S., and Pühler, A. 1995. A biological containment system for Rhizobium meliloti based on the use of recombination-deficient ( $r e c A)$ strains. FEMS Microbiol. Ecol. 16:223-232.

Soto, M. J., Zorzano, A., García-Rodriguez, F. M., Mercado-Blanco, J., López-Lara, I. M., Olivares, J., and Toro, N. 1994. Identification of a novel Rhizobium meliloti nodulation efficiency $n f e$ gene homolog of Agrobacterium ornithine cyclodeaminase. Mol. Plant-Microbe Interact. 7:703-707.

Tobar, R.M., Azcón-Aguilar, C., Sanjuan, J., and Barea, J. M. 1996. Impact of a genetically modified Rhizobium strain with improved nodulation competitiveness on the early stages of arbuscular mycorrhiza formation. Appl. Soil Ecol. 4:15-21.

Toro, N. 1996. Nodulation competitiveness in the Rhizobium-legume symbiosis. World J. Microbiol. Biotechnol. 12:157-162. 